\title{
Relationship between chewing tobacco, smoking, consuming alcohol and cognitive impairment among older adults in India: a cross-sectional study
}

\author{
T. Muhammad, Manideep Govindu and Shobhit Srivastava* (1)
}

\begin{abstract}
Background: Physical aging increases the sensitivity to the effects of substance use, elevating the risk for cognitive impairment among older adults. Since studies on the association of substance use with cognitive ability in later years are scant in India, we aimed to explore the factors associated with cognitive impairment especially, alcohol consumption, smoking, and chewing tobacco later in life.

Methods: The present research used nationally representative data from Building a Knowledge Base on Population Aging in India (BKPAl) that was conducted in 2011, across seven states of India ( $N=9,453)$. Sample distribution along with percentage distribution was calculated for cognitive impairment over explanatory variables. For finding the association between cognitive impairment over explanatory variables, binary logistic regression models were estimated.

Results: About 16.5 percent of older adults in rural areas consumed smoked tobacco compared to 11.7 percent in urban areas. Nearly, 23.7 percent of rural older adults consumed smokeless tobacco in comparison to 16 percent in urban areas. Alcohol consumption was high among rural residents (7.9\%) than urban counterparts (6.7\%). The prevalence of cognitive impairment was $62.8 \%$ and $58 \%$ among older adults from rural and urban areas respectively. Older adults who smoked tobacco had a 24 percent significantly higher likelihood to have cognitive impairment with reference to older adults who did not smoke [OR: 1.24, Cl: 1.02-1.49]. Moreover, older adults who consumed alcohol had a 30 percent significantly higher likelihood to have cognitive impairment [OR: 1.02, 1.65]. It was also found that older adults who had smoked along with consuming alcohol were at risk of worse cognitive outcomes than those who neither smoke nor drink alcohol [OR: 1.56, Cl: 1.21-2.00] or consumed either of them unlike consuming smokeless tobacco only.
\end{abstract}

Conclusion: The encouragement of older people to stop smoking and smokeless tobacco use could be considered as part of a strategy to reduce the incidence of cognitive impairment. Further, appropriate measures should be taken for the detection of early stages of cognitive decline in older individuals and efforts should be made to improve the availability and quality of care for dementing older adults.

\footnotetext{
* Correspondence: shobhitsrivastava889@gmail.com

International Institute for Population Sciences, 400088 Mumbai, Maharashtra, India
} 


\section{Background}

The longer people live, the greater the risk of aging biology in determining both length and quality of life [1]. Older adults are placed at increased risk of substance use disorders due to environmental and social factors associated with aging [2, 3]. Similarly, physical aging and commonly used medications can result in increased sensitivity to the effects of substance use, elevating the risk for cognitive impairment [4-7].

In 2016, Alzheimer's disease and other dementias appeared for the first time as ranked fifth noncommunicable disease in the World Health Organization (WHO) top 10 causes of death globally [8]. A systematic review revealed that the proportion of dementia that is characterized as the severe decline in cognitive functioning and the prevalence of cognitive impairment is increasing in developing countries [9]. Moreover, the study observed that although it is difficult to separate the effects of normal aging from that of disease, it appears that intellectual decline in some cognitive domains is an inevitable consequence of aging [10]. Further, a dearth of literature showed that the link between substance use disorders and objective cognitive outcomes has been well covered [11-13]. However, whether or not alcohol use is associated with cognition among older individuals is an unresolved issue.

\section{Alcohol drinking and tobacco use in later life}

The study found that heavy alcohol consumption in older adults has been associated with a faster decline in cognition in late middle age, particularly in men [14]. At the same time, evidence shows that consuming alcohol has a positive effect on several health outcomes. Interestingly, studies from developed as well as developing countries have shown that consuming alcohol at a mild to moderate level acts as a protective factor against cognitive decline among older adults, compared to excessive consumption of alcohol [15-17]. Although a number of studies have shown inconsistent results, light to moderate alcohol use among older people in some studies appeared to reduce the risk of dementia and Alzheimer's disease [18-20]. However, researchers have found that alcohol had a negative cognitive impact, even at moderate levels [21]. Many authors have argued about the causal role of substance use in exacerbating psychotic reactions [22]. Further, the study also showed that in psychotic patients, alcohol drinking and smoking may aggravate thought disturbances [23-25]. Similarly, as a result of increased alcohol dependence, the family life is harmed and had a strained relationship with other family members as well as with their neighbour's due to inappropriate behavior [26, 27] that may have led to poor health outcomes including cognition. Besides, a history of alcohol dependence, even when it is not associated with current heavy alcohol use, can also be associated with persistent cognitive impairment [28].

Furthermore, a recent study found that between 2010 and 2017, alcohol consumption in India increased by 38 percent from 4.3 to 5.9 liters per adult per year [29]. On the other hand, according to Global Adults Tobacco Survey India (GATS India, 2016-2017), in India, 41.4 percent of adults aged 65 years and older currently use tobacco (smoked and/or smokeless tobacco) [30]. The study also observed that tobacco use has been socially accepted among adults and older adults in most Indian societies and is a source of social interaction and recreational pursuit [31]. Unlike western countries, India has a higher number of smokeless tobacco consumers in comparison to smokers. Studies in India showed that smoking and chewing tobacco significantly correlated with the prevalence of coronary heart disease and hypertension and higher quality of life could be achieved by avoiding such habits [32, 33]. It is also demonstrated that tobacco use arguably accounts for far more medical disability and mortality in the older population than abuse of all other substances combined [2]. These findings serve to determine the effects of regular smoking on adverse health outcomes. Although daily smoking seemed to be associated with increased failures [34], it is difficult to disentangle the effects of cumulative use of tobacco over a lifetime on cognitive ability among older adults.

There is also great variation in levels of alcohol consumption, smoking and chewing tobacco and substance disorders across different demographic and socioeconomic groups in India. For instance, a comparative study of Bangladesh, India, and Nepal revealed that the prevalence of smokeless tobacco is higher among adult men in India than among women [35]. Previous work on mental, neurological and substance use disorders has posited that low education and poverty were associated with a higher occurrence of dementia in both China and India [36]. Again, people from lowest levels of socioeconomic groups were prone to report higher rates of smoking than their counterparts [37-39]. Study also suggests that populations with the poorest income status who increasingly purchase smokeless tobacco make use of scarce resources available with them and may have important indirect effects on their overall health [40]. Additionally, as a result of huge socioeconomic variations in the population, a recent study in India also found that several socio-economic and health factors such as increasing age, no schooling, and bedridden status for the past six months were significantly associated with higher cognitive impairment among the older population [41].

However, studies on the association of substance use with cognitive impairment among older adults are scant, 
especially in a country with low educational attainment. Thus, we aimed to explore the factors associated with the decline in cognitive functioning especially, alcohol consumption, smoking, and chewing tobacco. The study also sought to determine whether smoking/chewing tobacco and alcohol consumption together are associated with greater impairments in cognitive functioning in old age. The study hypothesizes that:

(1) Chewing tobacco, alcohol consumption, and smoking are positively associated with cognitive impairment in an aging population.

(2) There is a significant interaction between smoked/ smokeless tobacco use and alcohol consumption on cognitive impairment among older adults.

\section{Methods}

\section{Data}

The present research used data from Building a Knowledge Base on Population Aging in India (BKPAI) which was a nationally representative data was conducted in 2011, across seven states of India [42]. The survey was sponsored by the Tata Institute for Social Sciences (TISS), Mumbai, and Institute for Economic Growth (IEG), Delhi, Institute for Social and Economic Change (ISEC), and UNFPA (United Nations Population Fund), New Delhi. The survey gathered information on various socio-demographic, economic and health aspects of aging among households of those aged 60 years and above. The data from all the seven states were collected which represents the various regions of India. The states of Punjab and Himachal represent the northern part, Kerala and Tamil Nadu represent the southern part, Orissa and West Bengal represent the eastern part, and Maharashtra represents the western part of the country.

Being the survey of the older adults, the sample size was equally split between urban and rural areas, irrespective of the proportion of the urban and rural population. Eighty Primary Sampling Units (PSU) (villages or urban wards) - 40 urban and an equal number of rural - with 16 households per Primary Sampling Unit (PSU) having an older person were covered in the survey [42]. In all, 9850 older adults were interviewed from 8329 households aged 60 years and above. The sample included for the analysis after dropping the missing data (397 older adults) and outliers was 9453 older adults.

\section{Variable description \\ Outcome variable}

Cognitive impairment was measured by the number of words recalled. To measure the cognitive impairment, a scale of 0 to 10 was prepared. Higher score represents lower cognitive impairment and vice-versa. The words used for testing cognitive impairment were Bus, House,
Chair, Banana, Sun, Bird, Cat, Saree, Rice, and Monkey. Five or more words were recoded as 0 "low" representing lower cognitive impairment and a score of four or less was recoded as 1 "high" representing higher cognitive impairment [43-45]. High cognitive impairment represents cognitive disability among older adults in the present study. Place of Residence was recoded as rural and urban. The study was stratified into rural and urban place of residence. However, during multivariate analysis place of residence was used as a control variable to see the adjusted effects.

\section{Explanatory variable}

There were three main explanatory variables for the study. 1. Smoking tobacco was recoded as 0 "no" and 1 "yes". 2. Chewing tobacco was recoded as 0 "no" and 1 "yes". 3. Alcohol consumption was recoded as 0 "no" and 1 "yes". The questions assessed 'ever use of smoking tobacco', 'chewing tobacco' and 'alcohol consumption'.

Age was recoded as 60-69, 70-79, and $80+$ years [46]. Sex was recoded as Men and Women. Educational status was recoded as no schooling, below five years of schooling, 6-10 years of schooling, and 11 and above years of schooling. Working status was recoded as "yes", "no" and "retired". Marital status was recoded as currently in union and not in union "included never married, widowed, divorced and separated" [47]. Five questions for community involvement were asked and were used to create a variable to measure social capital. The score developed ranges from 0 to 5 and a score of 1 to 5 was recoded as 0 "community involvement" a score of 0 was recoded as 1 "no community involvement". The other question "do you have someone you can trust and confide in?" was recoded into binary form as 0 "yes" and 1 "no" [47]. Living arrangement was recoded as "others", "living alone and with spouse" [47]. Self-rated health had a scale of 1 to 5 "poor to excellent" and was recoded as 0 "good" (representing good, very good, and excellent) and 1 "poor" (representing poor or fair) [44, 47]. Chronic morbidity was recoded as 0 "no" and 1 "yes". Ability to do activities of daily living had a scale of 0 to 6 where it represents higher the score, higher the independence. A score of was recoded as 0 "high" which represents complete independence and 5 and less was recoded as 1 "low" which represents not completely independent to do activities of daily living (Cronbach Alpha: 0.93) [48]. Ability to do instrumental activities of daily living had a scale of 0 to 8 representing higher the score, higher the independence. A score of $6+$ was recoded as 0 "high" representing high IADL and a score of 5 and less was recoded as 1 "low" representing low IADL [42, 44, 46]. The International Classification of Functioning, Disability, and Health (ICF) proposed the framework on which ADL and IADL were calculated. The Activities of Daily 
Living (ADL) is an umbrella term relating to self-care, comprising those activities that people undertake routinely in their everyday life. The activities can be subdivided into personal care or ADL and domestic and community activities or Instrumental ADL (IADL). The ADL and IADL have emerged as the most common approaches in empirical assessments of functionality among the older adults and are considered to be befitting to the ICF framework [49].

Caste was recoded as non-Scheduled Caste/Scheduled Tribes and Scheduled Caste/Scheduled Tribes (SC/ST) [50]. Religion was recoded as Hindu, Muslim, and others. Wealth status was computed using 30 household assets and was divided into 5 quintiles as poorest, poorer, middle, richer, richest. Then the wealth index was divided as poor "poorest/poorer", middle and rich "richer/richest" [42]. Data for seven states was available in the data as mentioned in the data section.

\section{Statistical analysis}

Using STATA 14 [51] sample distribution along with percentage distribution was calculated for cognitive impairment over explanatory variables. For finding the association between cognitive impairment over explanatory variables binary logistic regression model [52] was used. The outcome variable was cognitive impairment coded as "low (0) and high (1)" and the main explanatory variables were consumption of tobacco (smoking and chewing) and consumption of alcohol.

The binary logistic regression model is usually put into a more compact form as follows:

$$
\operatorname{Logit}[\mathrm{P}(\mathrm{Y}=1)]=\beta_{0}+\beta * X+\epsilon
$$

The parameter $\beta_{0}$ estimates the log odds of the cognitive impairment for the reference group, while $\beta$ estimates the maximum likelihood, the differential log odds of the cognitive impairment associated with set of predictors $\mathrm{X}$, as compared to the reference group and $\epsilon$ represents the residual in the model.

The multivariate analysis had four models to explain the unadjusted and adjusted estimates. Model-1 was used to provide the independent effect of smoking tobacco, chewing tobacco, and alcohol consumption on cognitive impairment of older adults. Model-2 was an adjusted model (full-effect model) providing adjusted estimates. The model was adjusted for socio-economic and background characteristics. Model-3, Model-4, and Model-5 provide interaction effects $[45,53,54]$ for those who smoke tobacco and consume alcohol; chew tobacco and consume alcohol and who smoke and chew tobacco on cognitive impairment among older adults.

\section{Results}

Table 1 represents the socio-economic profile of older adults in India. About 16.5 percent of older adults consumed smoked tobacco in reference to 11.7 percent in urban areas. Nearly, 23.7 percent of older adults consumed chewing tobacco in comparison to 16 percent in urban areas.

About 11.4 percent of older adults were in the category of 80 + year's age group in rural areas and urban areas the percentage was 9.3 percent. Nearly 57.8 percent of older adults in rural areas had no education in comparison to 32 percent in urban areas. About 6.1 percent of older adults in rural areas were retired in reference to $15.3 \%$ in urban areas. Nearly, 38.1 percent of older adults in rural areas were not in a marital union in comparison to 43.2 percent in urban areas. About 22 percent of older adults from rural areas had no community involvement whereas the proportion was low in urban residents (17.7\%). Nearly, 18.6 percent and 13 percent of older adults from rural and urban areas had no trust over some someone respectively. Nearly, 23.2 percent of older adults lived alone/with the spouse in rural areas whereas the proportion was low for urban residents (19.5\%). About, 56.8 percent and 51.5 percent of older adults from rural and urban areas reported poor self-rated health status respectively. About, 7.7 percent and 59.5 percent of older adults from rural areas and 6.6 percent and 48.6 percent of older adults from urban areas reported low ADL and IADL respectively.

Table 2 represented percentage distribution of cognitive impairment among older adults by their background characteristics [rural $(n=4944)$ and urban $(n=4509)]$. In urban areas, older adults who smoked tobacco had a higher prevalence of cognitive impairment (58.4\%). Older adults who consumed chewing tobacco had a higher prevalence of cognitive impairment (rural-68.2\% and urban-62.3\%). Alcohol consumption had a positive association with cognitive impairment among older adults from urban areas (59.6\%).

Older adults aged 80 years and above had a higher prevalence of cognitive impairment (rural-79.1\% and urban $76.5 \%$ ). Older women had a higher prevalence of cognitive impairment (rural-69.2\% and urban-58.3\%). Older adults who had no education had the highest prevalence of cognitive impairment (rural-70.7\% and urban-69.8\%). Older adults who were reported as not working at the time of the survey had a higher prevalence of cognitive impairment (rural-68.7\% and urban-58.2\%). Being not in union (rural-72.3\% and urban-61.1\%), no community involvement (rural-70.0\% and urban-68.8\%), and no trust over someone (rural-70.1\% and urban$61.7 \%$ ) had a positive association with cognitive 
Table 1 Socio-economic profile of older adults in India

\begin{tabular}{|c|c|c|c|c|}
\hline \multirow[t]{2}{*}{ Variables } & \multicolumn{2}{|l|}{ Rural } & \multicolumn{2}{|l|}{ Urban } \\
\hline & Sample & Percentage & Sample & Percentage \\
\hline \multicolumn{5}{|l|}{ Smoking tobacco } \\
\hline No & 4128 & 83.5 & 3980 & 88.3 \\
\hline Yes & 816 & 16.5 & 529 & 11.7 \\
\hline \multicolumn{5}{|l|}{ Chewing tobacco } \\
\hline No & 3772 & 76.3 & 3788 & 84.0 \\
\hline Yes & 1172 & 23.7 & 721 & 16.0 \\
\hline \multicolumn{5}{|l|}{ Alcohol consumption } \\
\hline No & 4552 & 92.1 & 4207 & 93.3 \\
\hline Yes & 392 & 7.9 & 302 & 6.7 \\
\hline \multicolumn{5}{|l|}{ Age (years) } \\
\hline $60-69$ & 2954 & 59.7 & 3035 & 67.3 \\
\hline $70-79$ & 1429 & 28.9 & 1053 & 23.4 \\
\hline $80+$ & 562 & 11.4 & 421 & 9.3 \\
\hline \multicolumn{5}{|l|}{ Sex } \\
\hline Men & 2366 & 47.9 & 2077 & 46.1 \\
\hline Women & 2578 & 52.1 & 2432 & 53.9 \\
\hline \multicolumn{5}{|l|}{ Education } \\
\hline No education & 2858 & 57.8 & 1443 & 32.0 \\
\hline Below 5 years & 1026 & 20.8 & 892 & 19.8 \\
\hline $6-10$ years & 859 & 17.4 & 1643 & 36.4 \\
\hline $11+$ years & 201 & 4.1 & 531 & 11.8 \\
\hline \multicolumn{5}{|l|}{ Working status } \\
\hline Yes & 1299 & 26.3 & 835 & 18.5 \\
\hline No & 3342 & 67.6 & 2983 & 66.2 \\
\hline Retired & 303 & 6.1 & 691 & 15.3 \\
\hline \multicolumn{5}{|l|}{ Marital Status } \\
\hline Currently in Union & 3060 & 61.9 & 2561 & 56.8 \\
\hline Not in Union & 1884 & 38.1 & 1948 & 43.2 \\
\hline \multicolumn{5}{|l|}{ Community involvement } \\
\hline Yes & 3857 & 78.0 & 3710 & 82.3 \\
\hline No & 1087 & 22.0 & 799 & 17.7 \\
\hline \multicolumn{5}{|l|}{ Trust over someone } \\
\hline Yes & 4023 & 81.4 & 3922 & 87.0 \\
\hline No & 921 & 18.6 & 587 & 13.0 \\
\hline \multicolumn{5}{|l|}{ Living arrangement } \\
\hline Others & 3799 & 76.9 & 3631 & 80.5 \\
\hline Living alone/with spouse & 1145 & 23.2 & 878 & 19.5 \\
\hline \multicolumn{5}{|l|}{ Self-rated health } \\
\hline Good & 2138 & 43.3 & 2188 & 48.5 \\
\hline Poor & 2806 & 56.8 & 2321 & 51.5 \\
\hline \multicolumn{5}{|l|}{ Chronic morbidity } \\
\hline No & 1700 & 32.4 & 1691 & 37.5 \\
\hline Yes & 3244 & 65.6 & 2819 & 62.5 \\
\hline
\end{tabular}


Table 1 Socio-economic profile of older adults in India (Continued)

\begin{tabular}{|c|c|c|c|c|}
\hline \multirow[t]{2}{*}{ Variables } & \multicolumn{2}{|l|}{ Rural } & \multicolumn{2}{|l|}{ Urban } \\
\hline & Sample & Percentage & Sample & Percentage \\
\hline \multicolumn{5}{|l|}{ ADL } \\
\hline High & 4565 & 92.3 & 4209 & 93.4 \\
\hline Low & 379 & 7.7 & 300 & 6.6 \\
\hline \multicolumn{5}{|l|}{ IADL } \\
\hline High & 2002 & 40.5 & 2318 & 51.4 \\
\hline Low & 2942 & 59.5 & 2191 & 48.6 \\
\hline \multicolumn{5}{|l|}{ Caste } \\
\hline Non-SC/ST & 3493 & 70.7 & 3700 & 82.1 \\
\hline $\mathrm{SC} / \mathrm{ST}$ & 1451 & 29.3 & 809 & 18.0 \\
\hline \multicolumn{5}{|l|}{ Religion } \\
\hline Hindu & 3954 & 80.0 & 3535 & 78.4 \\
\hline Muslim & 307 & 6.2 & 415 & 9.2 \\
\hline Others & 683 & 13.8 & 560 & 12.4 \\
\hline \multicolumn{5}{|l|}{ Wealth index } \\
\hline Rich & 1300 & 26.3 & 2401 & 53.3 \\
\hline Middle & 962 & 19.5 & 1086 & 24.1 \\
\hline Poor & 2681 & 54.2 & 1020 & 22.6 \\
\hline \multicolumn{5}{|l|}{ States } \\
\hline Himachal Pradesh & 978 & 19.8 & 133 & 3.0 \\
\hline Punjab & 672 & 13.6 & 657 & 14.6 \\
\hline West Bengal & 528 & 10.7 & 692 & 15.4 \\
\hline Orissa & 908 & 18.4 & 308 & 6.8 \\
\hline Maharashtra & 658 & 13.3 & 799 & 17.7 \\
\hline Kerala & 651 & 13.2 & 779 & 17.3 \\
\hline Tamil Nadu & 548 & 11.1 & 1140 & 25.3 \\
\hline Total & 4944 & 100.0 & 4509 & 100.0 \\
\hline
\end{tabular}

ADL Activities of Daily Living; IADL Instrumental Activities of Daily Living; SC/ST Scheduled Caste/Scheduled Tribe

impairment among older adults. Older adults who reported poor self-rated health (rural-70.5\% and urban$63.7 \%$ ), chronic morbidity (rural-66.27 and urban-59.41), low ADL (rural-85.2\% and urban-82.6\%) and low IADL (rural-70.4\% and urban-59\%) had higher prevalence of cognitive impairment. Older adults from SC/ST category (rural-68.4\% and urban-63.1\%), Muslim religion (rural$69.6 \%$ and urban-60.5\%) and poor wealth status (rural$68.8 \%$ and urban-64.3\%) had high prevalence of cognitive impairment. Older adults from West Bengal had the highest cognitive impairment (rural-83.6\% and urban-78.5\%). Overall, older adults from rural areas had higher cognitive impairment than older adults from urban areas.

Table 3 provides logistic regression estimates for cognitive impairment among older adults in India. Model-1 was an unadjusted model which reveals that older adults who smoked tobacco had a 15 percent significantly higher likelihood to have cognitive impairment compared to older adults who did not smoke [OR: 1.15, CI: 1.01-1.31]. Similarly, older adults who chew tobacco had a 62 percent significantly higher likelihood to have cognitive impairment in comparison to older adults who do not chew tobacco [OR: 1.62, CI: 1.46-1.80].

Model-2 represents the full effect estimates. In here it was revealed that older adults who smoked tobacco had a 24 percent significantly higher likelihood to have cognitive impairment with reference to older adults who did not smoke [OR: 1.24, CI:1.02-1.49]. In this model interestingly, it was found that older adults who consumed alcohol had a 30 percent significantly higher likelihood to have cognitive impairment in comparison to older adults who did not consume alcohol [OR:1.02, 1.65]. Additionally, older adults 80 years and above [OR: 1.97, 
Table 2 Percentage distribution of cognitive impairment by background characteristics among older adults in India

\begin{tabular}{|c|c|c|c|c|}
\hline \multirow[t]{2}{*}{ Variables } & \multicolumn{2}{|c|}{ Rural } & \multicolumn{2}{|c|}{ Urban } \\
\hline & $\%$ & $\overline{p<0.05}$ & $\%$ & $p<0.05$ \\
\hline Smoking tobacco & & & & * \\
\hline No & 62.9 & & 51.2 & \\
\hline Yes & 62.4 & & 58.4 & \\
\hline Chewing tobacco & & * & & * \\
\hline No & 61.2 & & 50.1 & \\
\hline Yes & 68.2 & & 62.3 & \\
\hline Alcohol consumption & & & & * \\
\hline No & 63.2 & & 51.5 & \\
\hline Yes & 58.7 & & 59.6 & \\
\hline Age (years) & & * & & * \\
\hline $60-69$ & 56.4 & & 44.8 & \\
\hline 70-79 & 69.7 & & 63.2 & \\
\hline $80+$ & 79.1 & & 76.5 & \\
\hline Sex & & * & & * \\
\hline Men & 55.9 & & 44.7 & \\
\hline Women & 69.2 & & 58.3 & \\
\hline Education & & * & & * \\
\hline No education & 70.7 & & 69.8 & \\
\hline Below 5 years & 64.0 & & 61.4 & \\
\hline $6-10$ years & 42.7 & & 37.9 & \\
\hline $11+$ years & 30.7 & & 31.8 & \\
\hline Working status & & * & & * \\
\hline Yes & 54.0 & & 49.1 & \\
\hline No & 68.7 & & 58.2 & \\
\hline Retired & 35.5 & & 29.1 & \\
\hline Marital Status & & * & & * \\
\hline Currently in Union & 57.0 & & 45.1 & \\
\hline Not in Union & 72.3 & & 61.1 & \\
\hline Community involvement & & * & & * \\
\hline Yes & 60.8 & & 48.4 & \\
\hline No & 70.0 & & 68.8 & \\
\hline Trust over someone & & * & & * \\
\hline Yes & 61.2 & & 50.6 & \\
\hline No & 70.1 & & 61.7 & \\
\hline Living arrangement & & * & & * \\
\hline Others & 63.9 & & 55.3 & \\
\hline Living alone/with spouse & 59.2 & & 38.6 & \\
\hline Self-rated health & & * & & * \\
\hline Good & 52.7 & & 39.6 & \\
\hline Poor & 70.5 & & 63.7 & \\
\hline Chronic morbidity & & * & & * \\
\hline No & 56.3 & & 39.7 & \\
\hline Yes & 66.3 & & 59.4 & \\
\hline
\end{tabular}


Table 2 Percentage distribution of cognitive impairment by background characteristics among older adults in India (Continued)

\begin{tabular}{|c|c|c|c|c|}
\hline \multirow[t]{2}{*}{ Variables } & \multicolumn{2}{|c|}{ Rural } & \multicolumn{2}{|c|}{ Urban } \\
\hline & $\%$ & $p<0.05$ & $\%$ & $p<0.05$ \\
\hline ADL & & * & & * \\
\hline High & 61.0 & & 49.9 & \\
\hline Low & 85.2 & & 82.6 & \\
\hline IADL & & * & & * \\
\hline High & 51.7 & & 45.4 & \\
\hline Low & 70.4 & & 59.0 & \\
\hline Caste & & * & & * \\
\hline Non-SC/ST & 60.5 & & 49.6 & \\
\hline SC/ST & 68.4 & & 63.1 & \\
\hline Religion & & * & & * \\
\hline Hindu & 62.8 & & 50.8 & \\
\hline Muslim & 69.6 & & 60.5 & \\
\hline Others & 59.9 & & 53.8 & \\
\hline Wealth index & & * & & * \\
\hline Rich & 50.7 & & 46.2 & \\
\hline Middle & 62.7 & & 53.6 & \\
\hline Poor & 68.8 & & 64.3 & \\
\hline States & & * & & * \\
\hline Himachal Pradesh & 55.2 & & 34.7 & \\
\hline Punjab & 58.9 & & 45.5 & \\
\hline West Bengal & 83.6 & & 78.5 & \\
\hline Orissa & 70.0 & & 63.5 & \\
\hline Maharashtra & 56.7 & & 52.4 & \\
\hline Kerala & 69.1 & & 61.0 & \\
\hline T amil Nadu & 49.2 & & 32.3 & \\
\hline Total & 62.8 & & 52.0 & \\
\hline
\end{tabular}

ADL Activities of Daily Living; IADL Instrumental Activities of Daily Living; SC/ST Scheduled Caste/Scheduled Tribe; *if $p<0.05$; \%: percentage

CI: 1.57-2.47], older women [OR: 1.26, CI: 1.08-1.46], respondents with no educational status [OR: 3.42, CI: 2.59-4.50] and older adults with no work [OR: $1.23, \mathrm{CI}$ : 1.06-1.42] had a significant positive association with cognitive impairment. Older adults who were not in the marital union had a 14 percent significantly higher likelihood to have cognitive impairment with reference to older adults who were in marital union [OR: 1.14, CI: 1.00-1.13]. Older adults who had no trust over someone had significantly higher odds for cognitive impairment in reference to older adults who had trust over someone [OR: 1.22, CI: 1.04-1.44]. Moreover, poor self-rated health [OR: 1.50, CI: 1.34-1.69], chronic morbidity [OR: 1.36; CI: 1.20-154] low ADL [OR: 1.97, CI: 1.492.59] and low IADL [OR: 1.27, CI: 1.12-1.44] were significantly associated with cognitive impairment among older adults. Older adults from poor wealth status had significantly higher odds for cognitive impairment than older adults from rich wealth status [OR: 1.6CI: 1.36, 1.9].

Model-3, model-4, and model-5 were the models with interaction effects. It was revealed that older adults who had a habit of smoking tobacco and consuming alcohol simultaneously were at brisk of worst cognitive outcomes i.e. those older adults had 56 percent significantly higher likelihood to have cognitive impairment than older adults who neither smoke tobacco nor drink alcohol [OR: 1.56, CI: 1.21-2.00]. Similarly, the results were for older adults who chew tobacco and consume alcohol simultaneously however the results were not significant [OR: 1.26, CI: 0.9-1.78]. Additionally, older adults who had a habit to smoke and chew tobacco had a $15 \%$ higher likelihood to have cognitive impairment than older adults who neither smoke nor chew tobacco [OR: 1.15; CI: 0.82-1.60]. 
Table 3 Logistic regression estimates for cognitive impairment by background characteristics among older adults in India

\begin{tabular}{|c|c|c|c|c|c|}
\hline Variables & $\begin{array}{l}\text { Model-1 } \\
\text { OR }(95 \% \mathrm{Cl})\end{array}$ & $\begin{array}{l}\text { Model-2 } \\
\text { OR }(95 \% \text { Cl) }\end{array}$ & $\begin{array}{l}\text { Model-3 } \\
\text { OR }(95 \% \text { Cl) }\end{array}$ & $\begin{array}{l}\text { Model-4 } \\
\text { OR }(95 \% \mathrm{Cl})\end{array}$ & $\begin{array}{l}\text { Model-5 } \\
\text { OR }(95 \% \mathrm{Cl})\end{array}$ \\
\hline \multicolumn{6}{|l|}{ Smoking tobacco } \\
\hline No & Ref. & Ref. & & Ref. & \\
\hline Yes & $1.15^{*}(1.01,1.31)$ & $1.24 *(1.02,1.49)$ & & $1.22 *(1.01,1.47)$ & \\
\hline \multicolumn{6}{|l|}{ Chewing tobacco } \\
\hline No & Ref. & Ref. & Ref. & & \\
\hline Yes & $1.62^{*}(1.46,1.8)$ & $0.93(0.80,1.08)$ & $0.92(0.81,1.08)$ & & \\
\hline \multicolumn{6}{|l|}{ Alcohol consumption } \\
\hline No & Ref. & Ref. & & & Ref. \\
\hline Yes & $0.89(0.75,1.06)$ & $1.30 *(1.02,1.65)$ & & & $1.28 *(1.01-1.63)$ \\
\hline \multicolumn{6}{|l|}{ Age (years) } \\
\hline $60-69$ & & Ref. & Ref. & Ref. & Ref. \\
\hline $70-79$ & & $1.47^{*}(1.28,1.69)$ & $1.47^{*}(1.29,1.69)$ & $1.47^{*}(1.29,1.69)$ & $1.47^{*}(1.29,1.69)$ \\
\hline $80+$ & & $1.97^{*}(1.57,2.47)$ & $1.95 *(1.55,2.45)$ & $1.95 *(1.55,2.45)$ & $1.95 *(1.55,2.45)$ \\
\hline \multicolumn{6}{|l|}{ Sex } \\
\hline Men & & Ref. & Ref. & Ref. & Ref. \\
\hline Women & & $1.26 *(1.08,1.46)$ & $1.26 *(1.08,1.46)$ & $1.25^{*}(1.08,1.45)$ & $1.25^{*}(1.08,1.45)$ \\
\hline \multicolumn{6}{|l|}{ Education } \\
\hline No education & & $3.42^{*}(2.59,4.5)$ & $3.41^{*}(2.59,4.5)$ & $3.42^{*}(2.59,4.5)$ & $3.42 *(2.59,4.5)$ \\
\hline Below 5 years & & $2.60 *(1.98,3.4)$ & $2.59^{*}(1.97,3.39)$ & $2.59 *(1.98,3.39)$ & $2.59^{*}(1.98,3.39)$ \\
\hline $6-10$ years & & $1.38^{*}(1.08,1.77)$ & $1.39 *(1.08,1.78)$ & $1.39^{*}(1.08,1.78)$ & $1.39 *(1.08,1.78)$ \\
\hline $11+$ years & & Ref. & Ref. & Ref. & Ref. \\
\hline \multicolumn{6}{|l|}{ Working status } \\
\hline Yes & & Ref. & Ref. & Ref. & Ref. \\
\hline No & & $1.23^{*}(1.06,1.42)$ & $1.24^{*}(1.07,1.43)$ & $1.23^{*}(1.06,1.43)$ & $1.23^{*}(1.06,1.43)$ \\
\hline Retired & & $0.89(0.71,1.13)$ & $0.90(0.71,1.14)$ & $0.90(0.71,1.14)$ & $0.90(0.71,1.14)$ \\
\hline \multicolumn{6}{|l|}{ Marital Status } \\
\hline Currently in Union & & Ref. & Ref. & Ref. & Ref. \\
\hline Not in Union & & $1.14^{*}(1,1.31)$ & $1.14(0.99,1.3)$ & $1.14(0.99,1.3)$ & $1.14(0.99,1.3)$ \\
\hline \multicolumn{6}{|l|}{ Community involvement } \\
\hline Yes & & Ref. & Ref. & Ref. & Ref. \\
\hline No & & $1.13(0.98,1.31)$ & $1.14(0.99,1.32)$ & 1.14(0.99,1.32) & $1.14(0.99,1.32)$ \\
\hline \multicolumn{6}{|l|}{ Trust over someone } \\
\hline Yes & & Ref. & Ref. & Ref. & Ref. \\
\hline No & & $1.22 *(1.04,1.44)$ & $1.22^{*}(1.03,1.43)$ & $1.22 *(1.03,1.43)$ & $1.22^{*}(1.03,1.43)$ \\
\hline \multicolumn{6}{|l|}{ Living arrangement } \\
\hline Others & & Ref. & Ref. & Ref. & Ref. \\
\hline Living alone/with spouse & & $0.94(0.81,1.09)$ & $0.93(0.81,1.08)$ & $0.93(0.81,1.08)$ & $0.93(0.81,1.08)$ \\
\hline \multicolumn{6}{|l|}{ Self-rated health } \\
\hline Good & & Ref. & Ref. & Ref. & Ref. \\
\hline Poor & & $1.50 *(1.34,1.69)$ & $1.49^{*}(1.32,1.67)$ & $1.49 *(1.32,1.67)$ & $1.49^{*}(1.32,1.67)$ \\
\hline \multicolumn{6}{|l|}{ Chronic morbidity } \\
\hline No & & Ref. & Ref. & Ref. & Ref. \\
\hline Yes & & $1.36^{*}(1.20,1.54)$ & $1.36^{*}(1.20,1.54)$ & $1.36^{*}(1.20,1.54)$ & $1.36^{*}(1.20,1.54)$ \\
\hline \multicolumn{6}{|l|}{$A D L$} \\
\hline High & & Ref. & Ref. & Ref. & Ref. \\
\hline
\end{tabular}


Table 3 Logistic regression estimates for cognitive impairment by background characteristics among older adults in India (Continued)

\begin{tabular}{|c|c|c|c|c|c|}
\hline Variables & $\begin{array}{l}\text { Model-1 } \\
\text { OR }(95 \% \mathrm{Cl})\end{array}$ & $\begin{array}{l}\text { Model-2 } \\
\text { OR }(95 \% \mathrm{Cl})\end{array}$ & $\begin{array}{l}\text { Model-3 } \\
\text { OR }(95 \% \text { Cl) }\end{array}$ & $\begin{array}{l}\text { Model-4 } \\
\text { OR }(95 \% \mathrm{Cl})\end{array}$ & $\begin{array}{l}\text { Model-5 } \\
\text { OR }(95 \% \mathrm{Cl})\end{array}$ \\
\hline Low & & $1.97 *(1.49,2.59)$ & $1.95 *(1.48,2.56)$ & $1.95^{*}(1.48,2.56)$ & $1.95 *(1.48,2.56)$ \\
\hline \multicolumn{6}{|l|}{ IADL } \\
\hline High & & Ref. & Ref. & Ref. & Ref. \\
\hline Low & & $1.27^{*}(1.12,1.44)$ & $1.26^{*}(1.11,1.43)$ & $1.26 *(1.11,1.43)$ & $1.26 *(1.11,1.43)$ \\
\hline \multicolumn{6}{|l|}{ Caste } \\
\hline Non-SC/ST & & Ref. & Ref. & Ref. & Ref. \\
\hline SC/ST & & $1.08(0.94,1.21)$ & $1.11(0.95,1.27)$ & $1.11(0.95,1.27)$ & $1.11(0.95,1.27)$ \\
\hline \multicolumn{6}{|l|}{ Religion } \\
\hline Hindu & & Ref. & Ref. & Ref. & Ref. \\
\hline Muslim & & $0.87(0.68,1.11)$ & $0.87(0.68,1.1)$ & $0.87(0.68,1.11)$ & $0.87(0.68,1.11)$ \\
\hline Others & & $1.14(0.94,1.38)$ & $1.13(0.93,1.36)$ & $1.13(0.93,1.36)$ & $1.13(0.93,1.36)$ \\
\hline \multicolumn{6}{|l|}{ Wealth index } \\
\hline Rich & & Ref. & Ref. & Ref. & Ref. \\
\hline Middle & & $1.38 *(1.18,1.61)$ & $1.38^{*}(1.18,1.61)$ & $1.38 *(1.18,1.61)$ & $1.38^{*}(1.18,1.61)$ \\
\hline Poor & & $1.6^{*}(1.36,1.9)$ & $1.58 *(1.34,1.86)$ & $1.58 *(1.34,1.86)$ & $1.58^{*}(1.34,1.86)$ \\
\hline \multicolumn{6}{|l|}{ Place of residence } \\
\hline Urban & & Ref. & Ref. & Ref. & Ref. \\
\hline Rural & & $1.02(0.9,1.14)$ & $1.02(0.9,1.15)$ & $1.02(0.9,1.15)$ & $1.02(0.9,1.15)$ \\
\hline \multicolumn{6}{|l|}{ States } \\
\hline Himachal Pradesh & & Ref. & Ref. & Ref. & Ref. \\
\hline Punjab & & $0.98(0.75,1.29)$ & $0.98(0.75,1.29)$ & $0.98(0.75,1.29)$ & $0.98(0.75,1.29)$ \\
\hline West Bengal & & $4.21 *(3.23,5.49)$ & $4.28 *(3.28,5.59)$ & $4.28^{*}(3.28,5.58)$ & $4.28 *(3.28,5.58)$ \\
\hline Orissa & & $1.61 *(1.26,2.06)$ & $1.63^{*}(1.27,2.08)$ & $1.63^{*}(1.28,2.08)$ & $1.63 *(1.28,2.08)$ \\
\hline Maharashtra & & $1.17(0.94,1.47)$ & $1.11(0.89,1.39)$ & $1.10(0.88,1.38)$ & $1.10(0.88,1.38)$ \\
\hline Kerala & & $2.29^{*}(1.81,2.9)$ & $2.35 *(1.85,2.97)$ & $2.34^{*}(1.85,2.96)$ & $2.34^{*}(1.85,2.96)$ \\
\hline Tamil Nadu & & $0.58^{*}(0.46,0.73)$ & $0.60 *(0.48,0.75)$ & $0.60 *(0.48,0.75)$ & $0.60 *(0.48,0.75)$ \\
\hline \multicolumn{6}{|c|}{ Smoking tobacco \# Alcohol consumption } \\
\hline No \# No & & & Ref. & & \\
\hline No \# Yes & & & $1.39(0.93,2.07)$ & & \\
\hline Yes \# No & & & $1.24^{*}(1.01,1.52)$ & & \\
\hline Yes \# Yes & & & $1.56^{*}(1.21,2)$ & & \\
\hline \multicolumn{6}{|c|}{ Chewing tobacco \# Alcohol consumption } \\
\hline No \# No & & & & Ref. & \\
\hline No \# Yes & & & & $1.28(0.96,1.7)$ & \\
\hline Yes \# No & & & & $0.92(0.78,1.08)$ & \\
\hline Yes \# Yes & & & & $1.26(0.9,1.78)$ & \\
\hline \multicolumn{6}{|c|}{ Smoking tobacco \# chewing tobacco } \\
\hline No \# No & & & & & Ref. \\
\hline No \# Yes & & & & & $0.90(0.77-1.08)$ \\
\hline Yes \# No & & & & & $1.17(0.95-1.43)$ \\
\hline Yes \# Yes & & & & & 1.15(0.82-1.60) \\
\hline
\end{tabular}

ADL Activities of Daily Living; IADL Instrumental Activities of Daily Living; SC/ST Scheduled Caste/Scheduled Tribe; *if $p<0.05$; Ref Reference; \#: Interaction; OR Odds Ratio; Cl Confidence Interval 


\section{Discussion}

The increased life expectancy led populations to use substances for longer until the compounding effect of substance and declining health leads to significant morbidity and mortality [55-57]. Similarly, smoking and chewing tobacco increase morbidity and result in bad health outcomes both among adults and older individuals $[58,59]$. In agreement with this, the present study also confirms the first hypothesis we tested that alcohol consumption and tobacco use are positively associated with cognitive impairment in later years.

The natural aging process brings notable cognitive challenges. And the cognitive impairment adversely affects wellbeing and is a strong predictor for chronic disease progression, and subsequent mortality [60]. On the other hand, while substance use generally declines in later adulthood, even small amounts of alcohol use can have serious consequences [61]. Our results suggest that risk factors for cognitive impairment among older adults include higher age and female sex, while a higher educational level, working status, community involvement are protective factors. Compared to the results from studies conducted in the urban population in India $[41,62,63]$, the rates of cognitive impairment in rural areas is substantially higher, considering that a rural location is an established risk factor for dementia in Asian countries [64-66]. Also, the regression results show that lower educational attainment has been linked to lower cognitive ability. More years of education translates into a greater cognitive reserve [63, 67]. Older men and women who are not currently in the marital union were found to have lower cognitive ability compared to those who are currently in the marital union. It is consistent with earlier studies suggesting that older adults in any stressful marital relationship or widowhood may be as likely to engage in health-related substance use behaviour's due to increased stress from loss of financial and/or emotional resources and face more severe cognitive impairments $[66,68]$.

Similar to the patterns of cognitive impairment observed in Western studies that found an association of cognitive functioning with chronic diseases and somatic comorbidities $[69,70]$, our results also found that cognitive impairment was significantly associated with chronic morbidity. In concordance with previous studies in India [41], our study also observed that a substantial proportion of older adults with higher functional ability (ADL and IADL) reported lower cognitive impairment supporting the concept of successful cognitive aging. A couple of cohort studies also showed a positive association between self-rated health and current cognitive function among older adults and found poor self-rated health as a predictor of higher cognitive impairment in the advanced ages [71, 72]. Furthermore, a recent study also found that older adults with chronic kidney disease and poor functional and general health status were at increased risk for cognitive impairment [73]. Consistently, the results of the current study showed that those who reported poor self-rated health had lower cognitive ability than their counterparts.

Our findings also support the notion that smoking or chewing tobacco seems to be a risk factor for cognitive impairment. Current analysis found that smokers were more likely to be cognitively impaired than never smokers after adjusting for control variables such as age, sex, education, working status, and other social and health-related variables. Further, this study also observed alcohol use is positively associated with cognitive impairment. This is consistent with the view that alcohol consumption and substance use, which contribute to vascular diseases [19], could increase the risk for dementia and cognitive impairment $[74,75]$. On the other hand, it is also likely that those individuals who maintain cognitive function may display more adaptive qualities and abstain from substance use. Besides, the potential confounding effects of depressive symptoms, prescriptive drugs, and other substances are not taken into consideration in the current analysis. And finally, we cannot say for certain that maintaining cognitive function is causally related to not smoking or not consuming alcohol.

In the final model with interaction analyses, a significant interaction effect between the use of smoked tobacco and alcohol consumption on cognitive impairment was found. The older adults who were smokers and consumed alcohol exhibited greater cognitive deficits than those only smokers or who only consumed alcohol. Surprisingly, the odds of cognitive impairment were higher among those who used smokeless tobacco and consumed alcohol than those who consumed alcohol only unlike those who used smokeless tobacco only. Hence, though the results were not significant, the interaction effect also showed that smokeless tobacco is a predictor of cognitive impairment if the person consumed alcohol and used smokeless tobacco or used any form of tobacco that is, smoked and smokeless tobacco jointly leading to the partial confirmation of our second hypothesis. However, different mechanisms may underlie the adverse effects of heavy drinking and the beneficial effects of light to moderate drinking, and such mechanisms may also partly explain why certain differences are reported in decline in cognitive ability in later years.

The study suffers from certain limitations that need to be mentioned. Firstly, the results cannot be generalized for present scenario as the survey was conducted in 2011. Secondly, seven states of India that represent different regions of India were accounted to provide the estimates at national level. Therefore, one should be 
cautious while generalizing it for pan India. Another limitation of the study is that since it was cross-sectional rather than longitudinal, the findings should be interpreted with caution and it cannot attribute a final direction to the relationship of substance use and cognitive impairment. Moreover, given the benefits of moderate alcohol consumption and the risks of excessive drinking and smoking, the interplay between alcohol use and other substance use and cognitive ability requires further study to formulate a more crystallized understanding of the effect of substance use on cognitive health outcomes $[16,19,76]$. Finally, the dose and duration and consumption patterns of alcohol and tobacco have not been taken into account in the current study.

However, apart from these limitations, the BKPAI survey is one of the limited surveys in the context of India which provides such reliable estimates at the national level. Moreover, it is one of the most recent surveys in the Indian scenario which provide estimates on substance use among older adults and also report their cognitive functioning ability.

\section{Conclusions}

The findings of this study have implications for societies that are aging and consuming alcohol or chewing/smoking tobacco. When planning geriatric health care for older adults, priority must be given to older-older, women, illiterate, and those older adults who are socially less involved and have poor health outcomes, as they are more vulnerable to impaired cognitive function. Thus, the encouragement of older people to stop using smoked and smokeless tobacco could be considered as part of a strategy to reduce the incidence of cognitive impairment.

Moreover, despite the benefit of earlier diagnosis is established [56], most of the older adults with cognitive impairment do not receive a diagnosis and if they do, it happens late in their disease course. Hence, appropriate measures should be taken for the detection of early stages of cognitive decline in older individuals and efforts should be made to improve the availability and quality of care for dementing older adults. Furthermore, the preventive measures for cognitive impairment should focus on countering the risk factors suggested by the current evidence such as alcohol drinking, smoking and chewing tobacco, and other factors too. And other measures also include increased taxation of tobacco products and bans on its advertisements. Meanwhile, more research is warranted to identify the modifiable risk factors for declining cognitive ability among older adults.

\section{Abbreviations}

OR: Odds Ratio; Cl: Confidence Interval; ADL: Activities of Daily Living; IADL: Instrumental Activities of Daily Living; SC: Scheduled Caste; ST: Scheduled Tribe

\section{Acknowledgements}

Not applicable.

\section{Authors' contributions}

The concept was drafted by MT; SS assisted in paper conceptualization and contributed to the analysis design. MT, SS and MG contributed to the comprehensive writing of the article. SS and MT reviewed and modified with the contributions to the original manuscript. All authors read and approved the final manuscript.

\section{Author's Information}

Muhammad T completed his M.Phil. in Population Studies and currently pursuing his Ph.D. in Population Studies at International Institute for Population Sciences, Mumbai, India. Areas of research include population ageing and health disparities among vulnerable populations.

Manideep Govindu completed his M.Phil. in Population Studies from International Institute for Population Sciences, Mumbai, India.

Shobhit Srivastava completed his M.Phil. in Population Studies and currently pursuing his Ph.D. in Population Studies at International Institute for Population Sciences, Mumbai. His area of interest is ageing and mental health issues among older adults, India.

\section{Funding}

Authors did not receive any funding to carry out this research.

\section{Availability of data and materials}

The study utilizes a secondary data which is available only on request from director@isec.ac.in or india.office@unfpa.org.

\section{Ethics approval and consent to participate}

Ethical approval for this study, involving secondary data analyses, has been obtained from the Ethics Committee in the University of Southampton. Informed written consent was obtained from each participant, who were ensured that data would remain confidential and used for research purposes only.

\section{Consent for publication}

Not applicable.

\section{Competing interests}

The authors declare that they have no competing interests.

Received: 23 October 2020 Accepted: 13 January 2021

Published online: 29 January 2021

\section{References}

1. Dong $X$, Milholland B, Vijg J. Evidence for a limit to human lifespan. Nature. 2016;538(7624):257-9.

2. Jilk TJ, Mumenthaler MS. Substance use disorders in the elderly. Handbook of Mental Health and Aging. INC; 2020. 185-210 p.

3. Lemke S, Schutte KK, Brennan PL, Moos RH. Sequencing the lifetime onset of alcohol-related symptoms in older adults: Is there evidence of disease progression? I Stud Alcohol. 2005;66(6):756-65.

4. Moos RH, Brennan PL, Schutte KK, Moos BS. High-risk alcohol consumption and late-life alcohol use problems. Am J Public Health. 2004;94(11):1985-91.

5. Patterson $T L$, Jeste DV. The potential impact of the baby-boom generation on substance abuse among elderly persons. Psychiatr Serv. 1999;50(9):1184-8.

6. Yuan Z, Dawson N, Cooper GS, Einstadter D, Cebul R, Rimm AA. Effects of alcohol-related disease on hip fracture and mortality: A retrospective cohort study of hospitalized medicare beneficiaries. Am J Public Health. 2001;91(7): 1089-93.

7. Rehm J, Probst C. Decreases of life expectancy despite decreases in noncommunicable disease mortality: The role of substance use and socioeconomic status. Eur Addict Res. 2018;24(2):53-9.

8. World Health Organization. The top 10 causes of death. 2019

9. Prince M, Bryce R, Albanese E, Wimo A, Ribeiro W, Ferri CP. The global prevalence of dementia: A systematic review and metaanalysis. Alzheimer's Dement. 2013;9(1):63-75.e2.

10. Rinn WE. Mental Decline in Normal Aging: A Review. J Geriatr Psychiatry Neurol. 1988;1(3):144-58. 
11. David AS, Zammit S, Lewis G, Dalman C, Allebeck P. Impairments in cognition across the spectrum of psychiatric disorders: Evidence from a Swedish conscript cohort. Schizophr Bull. 2008;34(6):1035-41.

12. Hidalgo Vicario MH, Rodríguez Hernández PR. DSM-5. manual diagnóstico y estadístico de los trastornos mentales. últimas novedades. Pediatria Integral. 2013;Vol. 17:461-2 p.

13. Mahoney AM, Dalby JT, King MC. Cognitive Failures and Stress. Psychol Rep. 1998;82(3):1432

14. Sabia S, Elbaz A, Britton A, Bell S, Dugravot A, Shipley M, et al. Alcohol consumption and cognitive decline in early old age. Neurology. 2014;83(5):476

15. Ganguli M, Vander Bilt J, Saxton JA, Shen C, Dodge HH. Alcohol consumption and cognitive function in late life: A longitudinal community study. Neurology. 2005;65(8):1210-7.

16. Chan KKK, Chiu KC, Chu LW. Association between alcohol consumption and cognitive impairment in Southern Chinese older adults. Int J Geriatr Psychiatry. 2010;25(12):1272-9.

17. Zanjani F, Downer BG, Kruger TM, Willis SL, Schaie KW. Alcohol effects on cognitive change in middle-aged and older adults. Aging Ment Heal. 2013; 17(1):12-23.

18. Weyerer S, Schäufele M, Wiese B, Maier W, Tebarth F, Van den bussche $H$, et al. Current alcohol consumption and its relationship to incident dementia: Results from a 3-year follow-up study among primary care attenders aged 75 years and older. Age Ageing. 2011:40(4):456-63.

19. Peters R, Peters J, Warner J, Beckett N, Bulpitt C. Alcohol, dementia and cognitive decline in the elderly: A systematic review. Age Ageing. 2008; 37(5):505-12.

20. Huang W, Qiu C, Winblad B, Fratiglioni L. Alcohol consumption and incidence of dementia in a community sample aged 75 years and older. Clin Epidemiol. 2002;55(10):959-64.

21. Topiwala A, Allan CL, Valkanova V, Zsoldos E, Filippini N. Moderate alcohol consumption as risk factor for longitudinal cohort study adverse brain outcomes and cognitive decline. Dtsch Medizinische Wochenschrift. 2017; 142(16):1190-2.

22. Colzato LS, Ruiz MJ, van den Wildenberg WPM, Hommel B. Khat use is associated with impaired working memory and cognitive flexibility. PLoS One. 2011;6(6):1-6.

23. O'Dell KM, Hannay HJ, Biney FO, Robertson CS, Tian TS. The effect of blood alcohol level and preinjury chronic alcohol use on outcome from severe traumatic brain injury in hispanics, anglo-caucasians, and african-americans. J Head Trauma Rehabil. 2012;27(5):361-9.

24. Schuckit MA. Alcohol-use disorders. Lancet. 2009;373(9662):492-501.

25. Broe GA, Creasey H, Jorm AF, Bennett HP, Casey B, Waite LM, et al. Health habits and risk of cognitive impairment and dementia in old age: $A$ prospective study on the effects of exercise, smoking and alcohol consumption. Aust N Z J Public Health. 1998:22(5):621-3.

26. Saxena S, Sharma RAJ, Maulik PK. Impact of alcohol use on poor families: a study from North India. 2003:8(June):78-84.

27. Ramanan W, Singh SK. A study on alcohol use and its related health and social problems in rural Puducherry, India. 2017:804-8.

28. Woods AJ, Porges EC, Bryant VE, Seider T, Gongvatana A, Kahler CW, et al. Current Heavy Alcohol Consumption is Associated with Greater Cognitive Impairment in Older Adults. Alcohol Clin Exp Res. 2016;28(3):163-70.

29. Manthey J, Shield KD, Rylett M, Hasan OSM, Probst C, Rehm J. Global alcohol exposure between 1990 and 2017 and forecasts until 2030: a modelling study. Lancet. 2019:393(10190):2493-502.

30. MoHFW G of I, TISS. Global Adult Tobacco Survey- Second round INDIA 2016-17 Report. 2018.

31. Goswami A, Reddaiah V, Kapoor S, Singh B, Dwivedi S, Kumar G. Tobacco and alcohol use in rural elderly Indian population. Indian J Psychiatry. 2005:47(4):192

32. Gupta R. Smoking, educational status \& health inequity in India. Indian J Med Res. 2006;(July):15-22.

33. Ramadas K, Sauvaget C, Thomas G, Fayette J, Thara S, Sankaranarayanan R. Effect of tobacco chewing, tobacco smoking and alcohol on all-cause and cancer mortality : A cohort study from Trivandrum, India. Cancer Epidemiol. 2015;34(4):405-12.

34. Wan L, Friedman BH, Boutros NN, Crawford HJ. Smoking status affects men and women differently on schizotypal traits and cognitive failures. Pers Individ Dif. 2008:44(2):425-35.

35. Sinha DN, Rizwan SA, Aryal KK, Karki KB, Zaman M, Gupta PC. Trends of Smokeless Tobacco use among Adults (Aged 15-49 Years) in Bangladesh, India and Nepal. 2015;(October).
36. Cheng HG, Shidhaye R, Charlson F, Deng F, Lyngdoh T, Chen S, et al. Social correlates of mental, neurological, and substance use disorders in China and India: a review. The Lancet Psychiatry. 2016;3(9):882-99.

37. Rani M, Bonu S, Jha P, Nguyen SN, Jamjoum L. Tobacco use in India: prevalence and predictors of smoking and chewing in a national cross sectional household survey. Tob Control. 2003;12(4):1-8.

38. Hajat A, Kaufman JS, Rose KM, Siddiqi A, Thomas JC. Do the wealthy have a health advantage? Cardiovascular disease risk factors and wealth. Soc Sci Med. 2010;71(11):1935-42.

39. Silva GA e., Valente JG, de Almeida LM, de Moura EC, Malta DC. Tobacco smoking and level of education in Brazil, 2006. J Hazard Mater. 2009:67(2):48-56.

40. Hosseinpoor AR, Parker LA, Tursan d'Espaignet E, Chatterji S. Social determinants of smoking in low- and middle-income countries: Results from the world health survey. PLoS One. 2011;6:5.

41. Konda PR, Sharma PK, Gandhi AR, Ganguly E. Correlates of Cognitive Impairment among Indian Urban Elders. J Gerontol Geriatr Res. 2018;7:6.

42. UNFPA. Report on the status of elderly in selected states of India: Building a knowledge base on ageing in India. New Delhi, India; 2012.

43. Skirbekk V, Loichinger $E$, Weber $D$. Variation in cognitive functioning as a refined approach to comparing aging across countries. Proc Natl Acad Sci U S A. 2012

44. Joe W, Perkins JM, Subramanian SV. Community involvement, trust, and health-related outcomes among older adults in India: A population-based, multilevel, cross-sectional study. Age Ageing. 2019

45. Srivastava S, Muhammad T. Violence and associated health outcomes among older adults in India: A gendered perspective. SSM - Popul Heal. 2020;12:100702

46. Srivastava S, Gill A. Untreated morbidity and treatment-seeking behaviour among the elderly in India: Analysis based on National Sample Survey 2004 and 2014. SSM - Popul Heal [Internet]. 2020 Feb;100557. Available from: https://linkinghub.elsevier.com/retrieve/pii/S2352827320300124.

47. Srivastava S, Chauhan S, Muhammad T, Simon DJ, Kumar P, Patel R, et al. Older adults' psychological and subjective well-being as a function of household decision making role: Evidence from cross-sectional survey in India. Clin Epidemiol Glob Heal. 2021 Apr 1;10.

48. Muhammad T, Srivastava S. Why Rotational Living Is Bad for Older Adults ? Evidence from a Cross- Sectional Study in India. J Popul Ageing. 2020; (October).

49. World Health Organization. Towards a Common Language for Functioning, Disability and Health ICF. International Classification. 2002.

50. Subramanian SV, Nandy S, Irving M, Gordon D, Smith GD. Role of socioeconomic markers and state prohibition policy in predicting alcohol consumption among men and women in India: a multilevel statistical analysis. Bull World Health Organ. 2005;019893(04).

51. StataCorp. Stata. Release 14. Statistical Software. College Station: StataCorp LP; 2015.

52. Osborne J, King JE. Binary Logistic Regression. In: Best Practices in Quantitative Methods. SAGE Publications, Inc.; 2011. p. 358-84.

53. Van Der Weele TJ, Knol MJ. A tutorial on interaction. Epidemiol Method. 2014;3(1):33-72

54. Chauhan S, Srivastava S, Kumar P, Patel R, Dhillon P, T Muhammad. Interaction of substance use with physical activity and its effect on depressive symptoms among adolescents. J Subst Use [Internet]. 2020;1-7. Available from: https://www.tandfonline.com/doi/abs/https://doi.org/10. 1080/14659891.2020.1851411. [cited 2021 Jan 5].

55. Kennedy GJ, Efremova I, Frazier A, Saba A. The Emerging Problems of Alcohol and Substance Abuse in Late Life. J Soc Distress Homeless. 1999:8(4):227-39.

56. Thomas VS, Rockwood KJ. Alcohol abuse, cognitive impairment, and mortality among older people. J Am Geriatr Soc. 2001;49(4):415-20.

57. Davies G, Elison S, Ward J, Laudet A. The role of lifestyle in perpetuating substance use disorder: The Lifestyle Balance Model. Subst Abus Treat Prev Policy. 2015;10(1):1-8.

58. Pandey A, Patni N, Sarangi S, Singh M, Sharma K, Vellimana AK, et al. Association of exclusive smokeless tobacco consumption with hypertension in an adult male rural population of India. Tob Induc Dis. 2009;5(1):1-5.

59. Banjare P, Pradhan J. Socio-economic inequalities in the prevalence of multi-morbidity among the rural elderly in Bargarh district of Odisha (India). PLoS One. 2014;9(6)

60. Yaffe K, Lindquist K, Vittinghoff E, Barnes D, Simonsick EM, Newman A, et al. The Effect of Maintaining Cognition on Risk of Disability and Death. J Am Geriatr Soc. 2010;23(1):1-7. 
61. Schulte MT, Hser YI. Substance use and associated health conditions throughout the lifespan. Public Health Rev. 2014;35(2):1-27.

62. Mohan D, lype T, Varghese S, Usha A, Mohan M. A cross-sectional study to assess prevalence and factors associated with mild cognitive impairment among older adults in an urban area of Kerala. South India BMJ Open. 2019;9:3.

63. Samuel R, McLachlan CS, Mahadevan U, Isaac V. Cognitive impairment and reduced quality of life among old-age groups in Southern Urban India: Home-based community residents, free and paid old-age home residents. Qjm. 2016;109(10):653-9.

64. Zhang $Y, X u Y$, Nie H, Lei T, Wu Y, Zhang L, et al. Prevalence of dementia and major dementia subtypes in the Chinese populations: A meta-analysis of dementia prevalence surveys, 1980-2010. J Clin Neurosci. 2012;19(10): $1333-7$.

65. Ji Y, Shi Z, Zhang Y, Liu S, Liu S, Yue W, et al. Prevalence of dementia and main subtypes in rural northern China. Dement Geriatr Cogn Disord. 2015; 39(5-6):294-302.

66. Sharma D, Mazta S, Parashar A. Prevalence of cognitive impairment and related factors among elderly: A population-based study. J Dr NTR Univ Heal Sci. 2013;2(3):171.

67. Ren L, Bai L, Wu Y, Ni J, Shi M, Lu H, et al. Prevalence of and risk factors for cognitive impairment among elderly without cardio-and cerebrovascular diseases: A population-based study in rural China. Front Aging Neurosci. 2018:10(MAR):1-8

68. Perkins JM, Lee HY, Lee JK, Heo J, Krishna A, Choi S, et al. Widowhood and Alcohol, Tobacco, and Other Drug Use among Older Adults in India. Journals Gerontol - Ser B Psychol Sci Soc Sci. 2018;73(4):666-74.

69. Millán-Calenti JC, Tubío J, Pita-Fernández S, González-Abraldes I, Lorenzo T, Maseda A. Prevalence of Cognitive Impairment: Effects of Level of Education, Age, Sex and Associated Factors. Dement Geriatr Cogn Disord. 2009;28(5):455-60.

70. Clodomiro A, Gareri P, Puccio G, Frangipane F, Lacava R, Castagna A, et al. Somatic comorbidities and Alzheimer's disease treatment. Neurol Sci. 2013; 34(9):1581-9.

71. Bond J, Dickinson ÆEHO, Matthews ÆFF, Jagger C, Brayne ÆC, Cfas ÆMRC Self-rated health status as a predictor of death, functional and cognitive impairment : a longitudinal cohort study. 2006;193-206

72. Kato K, Zweig R, Schechter CB, Verghese J, Barzilai N, Atzmon G Personality, self - rated health, and cognition in centenarians : Do personality and self - rated health relate to cognitive function in advanced age ? 2013;5(3):183-91.

73. Coppolino G, Bolignano D, Gareri P, Ruberto C, Andreucci M, Ruotolo G, et al. Kidney function and cognitive decline in frail elderly: two faces of the same coin? Int Urol Nephrol. 2018:50(8):1505-10.

74. Ivan CS, Seshadri S, Beiser A, Au R, Kase CS, Kelly-Hayes M, et al. Dementia after stroke: The Framingham study. Stroke. 2004;35(6):1264-8.

75. Panza F, Capurso C, D'Introno A, Colacicco AM, Frisardi V, Santamato A, et al. Vascular risk factors, alcohol intake, and cognitive decline. J Nutr Heal Aging. 2008;12(6):376-81

76. Patel V, Chatterii S, Chisholm D, Ebrahim S, Gopalakrishna G, Mathers C, et al. India: Towards Universal Health Coverage 3: Chronic diseases and injuries in India. Lancet. 2011;377(9763):413-28.

\section{Publisher's Note}

Springer Nature remains neutral with regard to jurisdictional claims in published maps and institutional affiliations.

Ready to submit your research? Choose BMC and benefit from:

- fast, convenient online submission

- thorough peer review by experienced researchers in your field

- rapid publication on acceptance

- support for research data, including large and complex data types

- gold Open Access which fosters wider collaboration and increased citations

- maximum visibility for your research: over $100 \mathrm{M}$ website views per year

At BMC, research is always in progress.

Learn more biomedcentral.com/submissions 